\title{
Brain Tuberculoma Management Challenges: A Series of 5 Cases
}

\author{
Khan RN ${ }^{1}$, Ahmed M T ${ }^{2}$, Asaduzzaman $\mathrm{SM}^{3}$, Ahmed M U4
}

Conflict of interest: There is no conflict of interest relevant to this paper to disclose.

Funding Agency: Was not funded by any institute or any group.

Contribution of Authors: Khan RN was Principal investigator, Ahmed MT help for protocol preparation, Asaduzzaman SM

help for data collection and Ahmed M U help for editorial formatting.

Copyright: @2019 Bang.JNS published by BSNS. This article is published under the creative commons CC-BY-NC license.

This license permits use distribution (https://creativecommons.orgf/ licences/by-nc/4-0/) reproduction in any medium, provided the original work is properly cited, and is not used for commercial purposes.

Received: 20 February, 2019

Accepted: 04 March, 2019

\begin{abstract}
Although intracranial tuberculomas are not rare \& account for a significant number of ICSOLs; Its clinical presentation \&neuro-radiological findings sometimes lead us to a diagnostic dilemma and management challenge. Here, in this case series we are presenting diagnostic as well as management challenges we've faced managing patients with tuberculoma at a tertiary care hospital of Bangladesh. Intracranial tuberculomas can be situated in various locations and can mimic any lesion.Extracranial involvement is not always common, clinical presentation \&neuroradiological findings sometimes not conclusive. Medical management indicated in almost all cases, as well as surgical intervention indicated in cases with neurological deficits \& mass effect.
\end{abstract}

Keywords: Brain Tuberculoma, intracranial TB, extrapulmonary TB

Bang. J Neurosurgery 2019; 9(1): 64-68

histological data. ${ }^{3,4}$ For this reason, especially in areas with high disease prevalence, an empirical trial of antituberculosis therapy containing potent drugs such as rifampicin (RMP) is advocated.[5] Only a high index of suspicion or a presumptive diagnosis based on typical clinical and neuro-image ûndings sufûces to warrant such treatment. For patients with neurological deficits, surgical intervention is recommended. ${ }^{6,7}$

Here, we are presenting 5 cases with tuberculoma we have managed in our hospital.

\section{Patients}

\section{Case 1.}

A 20 year old lady was admitted to our unit with headache \& vomiting for 2 months with history of several episodes of convulsion for 2 weeks. No H/O fever.

On examination, she was drowsy, had bilateral papilaedema and right hemiparesis. Her temperature

1. Dr. Md. Rashidoon Nabi Khan, Associate Professor \& Head, Department of Neurosurgery, SOMCH.

2. Dr. MostafaTaufiq Ahmed, Assistant Professor, Department of Neurosurgery, SOMCH

3. Dr. S M AsaduzzamanJuel, Resident Surgeon, Department of Neurosurgery, SOMCH.

4. Dr. Mesbah Uddin Ahmed, Assistant Registrar, Department of Neurosurgery, SOMCH

Address of the Correspondence: Dr. Mesbah Uddin Ahmed, Assistant Registrar, Department of Neurosurgery, SOMCH. E-mail: dr.mesbah.bd@gmail.com 
was $37^{\circ} \mathrm{C}$. ESR was $60 \mathrm{~mm}$ in $1^{\text {st }}$ hour. Chest radiograph was normal.

MRI of brain showed multiple hypo intense coalescence lesions with peripheral hyper intense rim on left parietal region with significant midline shift.

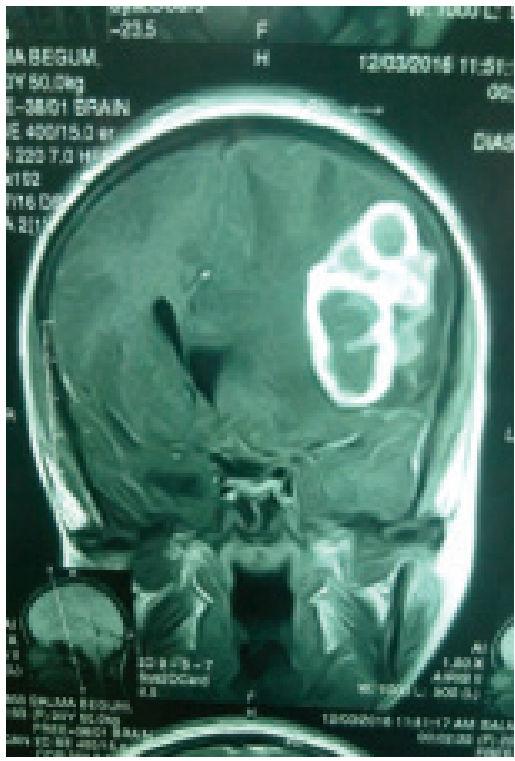

Patient underwent left parietal craniotomy with removal of the lesion. Histopathology confirmed as tuberculoma. Patient was treated with WHO recommended fixed dose combination anti-tubercular regimens for 18 months, in addition to oral steroids with tapering doses and anti-convulsants for 3 months.

On follow up visit after 3 months of starting antitubercular drugs, patient was completely symptomless living a normal life.

\section{Case 2.}

An 18 year old boy was admitted to our unit with headache \& vomiting for 7 months with history of several episodes of convulsion for 2 weeks. He had a history of recent contact with a PTB patient, but no H/ O fever.

On examination, he was conscious, had bilateral papilaedema. His temperature was $37^{\circ} \mathrm{C}$. ESR was $60 \mathrm{~mm}$ in $1^{\text {st }}$ hour. Chest radiograph was normal.

MRI of brain showed multiple hypo intense coalescence lesions with peripheral hyper intense rim on right occipital region.

Patient underwent right occipital craniotomy with removal of the lesion. Histopathology confirmed as tuberculoma. Patient was treated with WHO

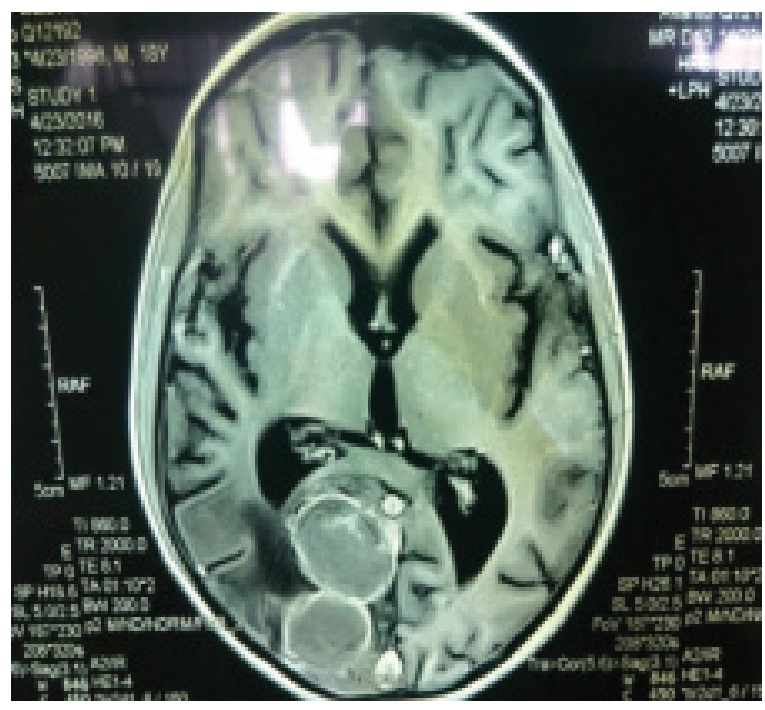

recommended fixed dose combination anti-tubercular regimens for 18 months, in addition to oral steroids with tapering doses and anti-convulsants for 3 months.

On follow up visit after 3 months of starting antitubercular drugs, patient was living a normal life with visual impairment

\section{Case 3.}

A 15 year old girl was admitted to our unit with headache \& vomiting for 4 months with right hemiparesis for 2 weeks.

On examination, she was conscious, alert \& oriented; had bilateral papilaedema and muscle power $3 / 5$ in right upper \& lower limbs, jerks normal, planter

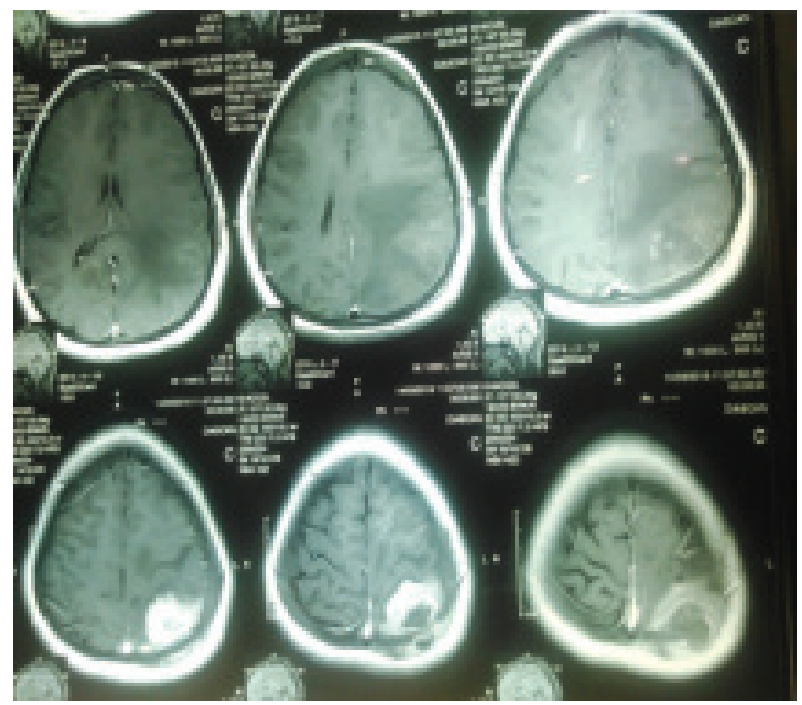


equivocal B/L. Her temperature was $37^{\circ} \mathrm{C}$. ESR was $45 \mathrm{~mm}$ in $1^{\text {st }}$ hour. Chest radiograph was normal.

MRI of brain showed an irregularhypo intense lesion with peripheral hyper intense rim on left parietal region with mild perilesionaloedema.

Patient underwent left parietal craniotomy with removal of the lesion. Histopathology confirmed as tuberculoma. Patient was treated with WHO recommended fixed dose combination anti-tubercular

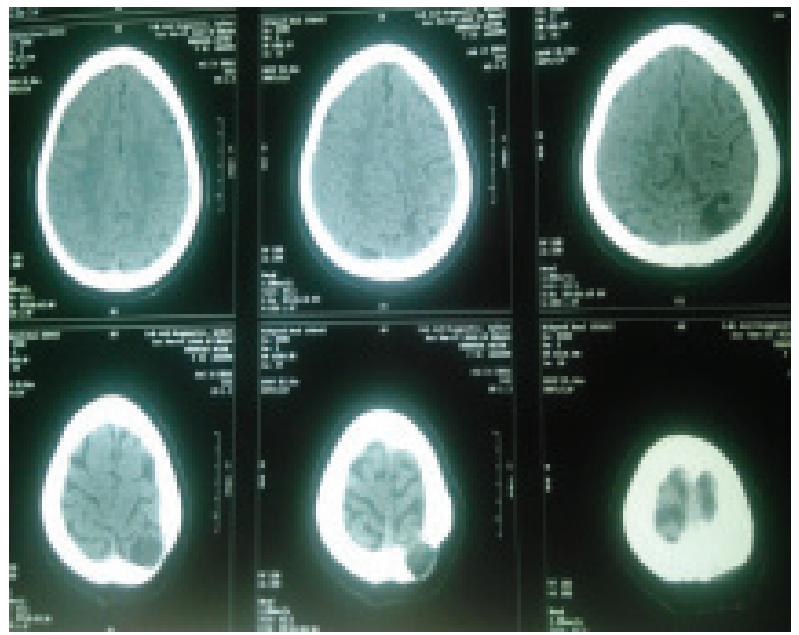

regimens for 18 months, in addition to oral steroids with tapering doses and anti-convulsants for 3 months.

Follow up CT scan of brain showed no persistence of old lesions as well as normal brain findings with evidence of surgery.

On follow up visit after 3 months of starting antitubercular drugs, patient was completely symptomless living an apparently normal life.

\section{Case 4.}

A 40 year old diabetic female was admitted to our unit with headache \& vomiting for 4 months with occasional focal convulsion for 3 weeks.

On examination, she was conscious, alert \& oriented; had bilateral papilaedema. Her temperature was $37.5^{\circ}$ C. ESR was $75 \mathrm{~mm}$ in $1^{\text {st }}$ hour. Chest radiograph was normal.

CT scan of brain showed an irregular hypo dense lesion with light peripheral hyper dense rim on left fronto-parietal region with mild perilesionaloedema.

Our clinical diagnosis wastuberculoma. Patient was empirically treated with WHO recommended fixed dose combination anti-tubercular regimens for 18

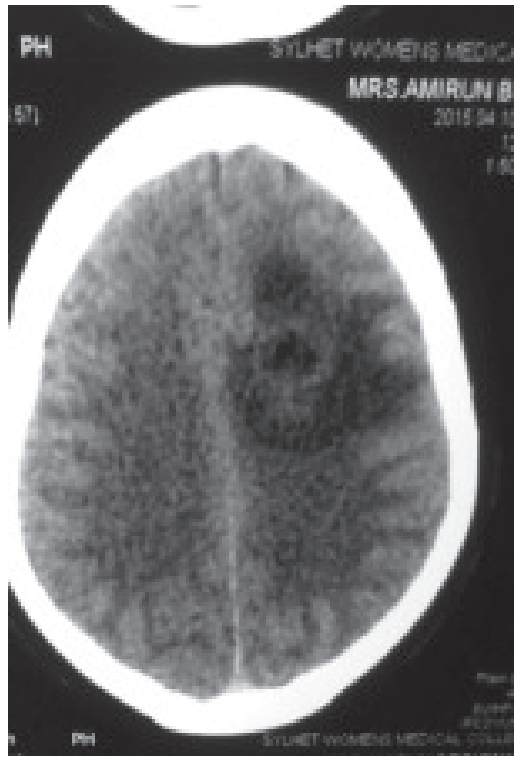

months, in addition to oral steroids with tapering doses and anti-convulsants for 3 months.

On follow up visit after 3 months of starting antitubercular drugs, patient was completely symptomless living an apparently normal life.

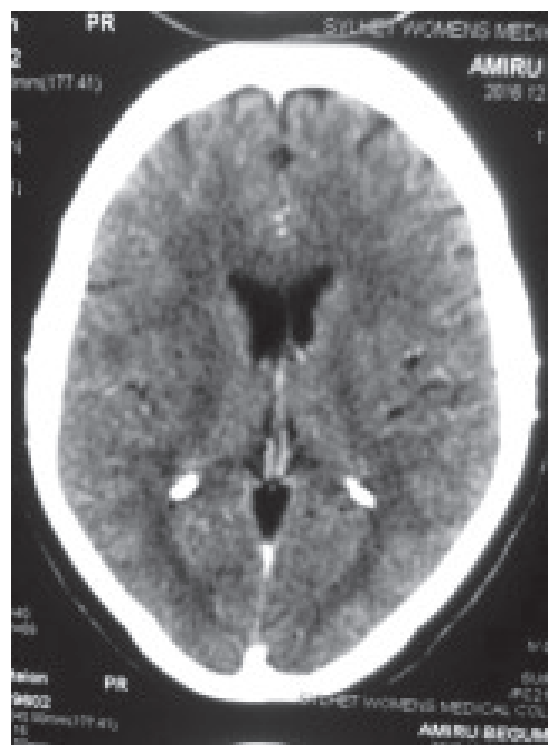

Follow up CT scan of brain showed no persistence of old lesions and normal brain CT findings.

\section{Case 5.}

A 16 year old girl was admitted to our unit with headache \& vomiting for 2 months with occasional focal convulsion for 1 week \& occasional abnormal behabior. 
On examination, she was conscious, alert \& oriented; had bilateral papilaedema. Her temperature was $37.5^{\circ}$ C. ESR was $80 \mathrm{~mm}$ in $1^{\text {st }}$ hour. Chest radiograph was normal.

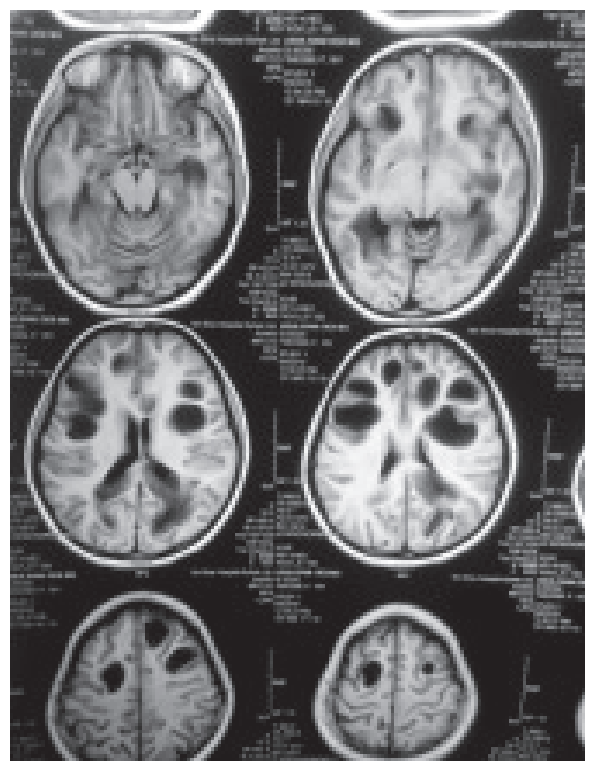

MRI of brain showed, multiple irregular hypo intense(in $\mathrm{T} 1 \mathrm{WI}$ ) \& hyper intense(in T2WI) lesions on bothfrontoparietal region with significant perilesionaloedema.

Our clinical diagnosis was tuberculoma. Patient was empirically treated with $\mathrm{WHO}$ recommended fixed dose combination anti-tubercular regimens for 18 months, in addition to oral steroids with tapering doses and anti-convulsants for 3 months.

On follow up visit after 3 months of starting antitubercular drugs, patient was symptomless living an apparently normal life with occasional abnormal hehabior.

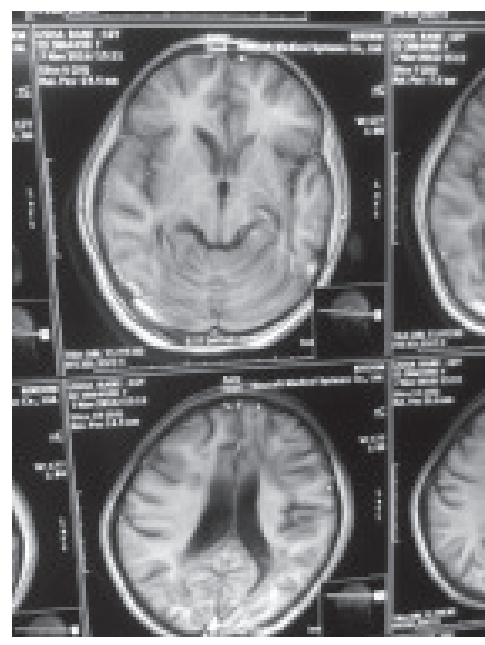

Follow up MRI of brain showed no persistence of old lesions as well as normal brain findings.

\section{Discussion:}

Tuberculosis is still a serious health problem in developing countries. There is also resurgence in developed countries due to human immunodeficiency virus (HIV), immigration and development of multi-drug resistant strains. ${ }^{1}$ Central nervous system involvement of tuberculosis is $10 \%$, and it may appear as tuberculous meningitis, tuberculoma, abscess or Pott's disease. ${ }^{8}$ Tuberculomas account for $10-30 \%$ of all intracranial masses in developing countries, and $0.5-2 \%$ in developed countries. ${ }^{2}$ They are commonly located in cerebral hemispheres and basal ganglia in adults, and in cerebellar hemispheres in children, due to the large blood supply to these areas. ${ }^{9}$ Rare locations, such as brainstem, cerebellopontine angle, and pituitary gland have also been reported. ${ }^{10}$

The clinical manifestations of brain tuberculoma are pleomorphic. Mainly related to individual differences in size and topography of lesion. Symptoms and signs of raised intra cranial pressure (ICP) are usual features and constitute headache, vomiting, blurring of vision, and papilloedema. Lateralising signs when the lesion is in cerebrum. Focal or generalized convulsions also. Signs and symptoms of meningitis i.e. neck stiffness and fever.

The presenting symptoms \& signs are summarized in Table 1.

\begin{tabular}{lc}
\hline Symptoms \& Signs & No of Patients (n) \\
\hline Headache & $05(100 \%)$ \\
Vomiting & $05(100 \%)$ \\
Convulsion & $04(80 \%)$ \\
Hemiparesis & $03(60 \%)$ \\
Papilloedema & $05(100 \%)$ \\
Fever & $01(20 \%)$ \\
\hline
\end{tabular}

Duration of symptoms at presentation varies from 2 to 7 months. None of the patients had extracranial TB. None had previous H/O PTB. But one patient had $\mathrm{H} / \mathrm{O}$ contact with PTB patient \& another patient was diabetic. Therewere no other systemic diseases among the patients of this series. In all cases HIV 1 \& 2 was negative, though sylhet is a known zone for a number of HIV infected patients.

In our cases, CT findings of the lesions presented as irregular hypo dense lesion with hyper dense rim 
enhancement (case 4). MRI findings revealed in rest of the cases as hypo intense lesion in T1WI \& hyper intense lesion in T2WI.

CT scans of a tuberculoma reveal an iso - to hyperdense lesion with varying contrast enhancement pattern. ${ }^{11}$ T1 weighted MRI demonstrates an iso - to - hypointense lesion. On T2-weighted images, it can appear as a hyperintense lesion or a hyperintense center surrounded by a hypointense rim. ${ }^{12}$ Therefore there are no pathognomonic radiological findings for a tuberculoma. There is also a recent paper regarding diffusion-weighted MRI and MRI spectroscopy in the diagnosis of tuberculoma, but it is concluded that these techniques are also unable to provide a specific characterization. ${ }^{13}$

Surgery was performed in 3 of the 5 cases, in order to relief the mass effect and also to establish a diagnosis. In all 3 cases; the lesions were completely removed. Histopathological examination of a tuberculoma reveals a necrotic caseous center surrounded by a capsule composed of fibroblasts, epitheloid cells, Langhans giant cells and lymphocytes. ${ }^{1}$ Biopsy of the lesion is essential for establishing a diagnosis. Recent papers also suggest stereotactic biopsy as an alternative to craniotomy, and diagnostic yields up to $85 \%$ have been reported. ${ }^{14}$

Mainstay of treatment in intracranial tuberculomas is medical. Surgery is reserved for large, solitary lesions with significant mass effect and unresponsive to medical treatment. ${ }^{2}$ Medical treatment consists of isoniazid, rifampin, and pyrazinamide for the initial 2 months, followed by only isoniazid and rifampin for the remaining time. ${ }^{8}$ Although the recommended duration of treatment is 12 months, shorter and longer courses are proposed. ${ }^{9}$

In our cases we advised our patients 18 months supervised regimens. We have follow up the patients monthly, as all the cases were common at $3^{\text {rd }}$ month follow up, we presented our follow up at $3^{\text {rd }}$ month in this literature. Addition of steroids to the regimen is advised, in order to prevent paradoxical expansion of the lesion during medical treatment. ${ }^{15}$ Overall mortality is $10 \% .12$

\section{Conclusion:}

In conclusion, intracranial tuberculomas can be situated in every location and can mimic any lesion. Pulmonary involvement is not always present and radiological studies are not conclusive. Biopsy is diagnostic and treatment is medical. Especially in the presence of risk factors, a high index of suspicion should be maintained. But in case of large, solitary lesions with significant mass effect, patients with neurological deficits \& unresponsive to medical management need surgical interventions.

\section{References:}

1. Hua SE, Clatterbuck RE, Stern BJ, Sampath P, Rhines LD. Sarcoidosis, tuberculosis, and xanthogranuloma In: Winn HR, editor. Youmans Neurological Surgery, Vol 1.3 of 4Philadelphia: Saunders 2004:1435-1447.

2. Artico M, De Caro GM, Carloia S, Salvati M, D'Ambrosio M, Delfini R. Advances in diagnosis, treatment, and prognosis of intracerebraltuberculomas in the last 50 years: Report of 21 cases. Neurochirurgie 1999; 45:129-133.

3. Bourchama A, Zuheir Al-Kawi M, et al. Brain biopsy in tuberculoma: the risks and beneûts. Neurosurgery 1991; 28: 405-409.

4. Jayasundar R, Raghunthan P, Banerji A K. Proton MRS similarity between central nervous system non-Hodgkin lymphoma and intractanialtuberculoma. MagnReson Imaging 1995; 13: 489-493.

5. Oscar H, Brutto D, Mosquera A. Brainstem tuberculoma mimicking glioma: the role of antituberculous drugs as a diagnostic tool. Neurology 1999; 52: 210-211.

6. Haddadian K, Rezaei O, Samadian M. Multiple Brain TuberculomasAnd Role Of Open Brain Biopsy: A Case Report And Review. Internet Jf Infect Dis. 2005;4(1).

7. Radmanesh F, Nejat F, ElKhashab M. Cerebral Infarction as the First Presentation of Tuberculosis in an Infant: A Case Report. J Microbiollmmunol Infect. 2010;43(3): 249-52.

8. Gropper MR, Schulder M, Sharan AD, Cho ES. Central nervous system tuberculosis: Medical management and surgical indications. SurgNeurol 1995;44:378-385.

9. Al Soub H, Al Alousi FS, Al-Khal AL. Tuberculoma of the cavernous sinus. Scand J Infect Dis 2001;33:868-870.

10. Ashkan K, Papadopoulos MC, Casey AT, Thompson DN, Jarvis S, Powell M, Thomas DGT. Sellartuberculoma: Report of two cases. ActaNeurochir (Wien) 1997;139:523-525.

11. Vengsarkar US, Pisipaty RP, Parekh B, Panchal VG, Shetty MN. Intracranial tuberculoma and the CT scan. J Neurosurg 1986;64:568-574.

12. Gupta RK, Jena A, Singh AK, Sharma A, Puri V, Gupta M. Role of magnetic resonance (MR) in the diagnosis and management of intracranial tuberculomas. ClinRadiol 1990;41:120-127.

13. Batra A, Tripathi RP. Diffusion-weighted magnetic resonance imaging and magnetic resonance spectroscopy in the evaluation of focal cerebral tubercular lesions. ActaRadiol 2004;45:679-688.

14. Mohanty A, Santosh V, Anandh B, Kolluri VR, Vasudev MK, Hegde T, Shankar SK. Diagnostic efficacy of stereotactic biopsies in intracranial tuberculomas. SurgNeurol 1999;52:252-258.

15. Reiser M, Fatkenheuer G, Diehl V. Paradoxical expansion of intracranial tuberculomas during chemotherapy. J Infect 1997; 35:88-90. 